Article

\title{
Extreme Ultraviolet Spectra of Few-Times Ionized Tungsten for Divertor Plasma Diagnostics
}

\author{
Joel Clementson ${ }^{1, *}$, Thomas Lennartsson ${ }^{2}$ and Peter Beiersdorfer ${ }^{1}$ \\ ${ }^{1}$ Physics Division, Lawrence Livermore National Laboratory, Livermore, CA 94550, USA \\ ${ }^{2}$ Lund Observatory, Lund University, SE-221 00 Lund, Sweden \\ * Author to whom correspondence should be addressed; E-Mail: joel.clementson@ gmail.com. \\ Academic Editor: Elmar Träbert
}

Received: 11 April 2015 / Accepted: 17 August 2015 / Published: 9 September 2015

\begin{abstract}
The extreme ultraviolet (EUV) emission from few-times ionized tungsten atoms has been experimentally studied at the Livermore electron beam ion trap facility. The ions were produced and confined during low-energy operations of the EBIT-I electron beam ion trap. By varying the electron-beam energy from around $30-300 \mathrm{eV}$, tungsten ions in charge states expected to be abundant in tokamak divertor plasmas were excited, and the resulting EUV emission was studied using a survey spectrometer covering 120-320 A. It is found that the emission strongly depends on the excitation energy; below $150 \mathrm{eV}$, it is relatively simple, consisting of strong isolated lines from a few charge states, whereas at higher energies, it becomes very complex. For divertor plasmas with tungsten impurity ions, this emission should prove useful for diagnostics of tungsten flux rates and charge balance, as well as for radiative cooling of the divertor volume. Several lines in the 194-223 A interval belonging to the spectra of five- and seven-times ionized tungsten (Tm-like W VI and Ho-like W VIII) were also measured using a high-resolution spectrometer.
\end{abstract}

Keywords: EBIT; tokamak divertor; plasma diagnostics; tungsten ions; EUV spectroscopy

\section{Introduction}

The increased usage of tungsten as a wall material in magnetic fusion devices has led to the need for accurate atomic data of tungsten for essentially all ionization stages [1,2]. In addition to the large influence on the plasma power balance, tungsten emission may provide diagnostic information from all parts of the plasma, from the hot core to the cooler edge and divertor regions. In a tokamak divertor, 
where most of the plasma-wall interactions take place, operating temperatures of the plasma volume must be low, $T_{e} \approx 1-150 \mathrm{eV}$. Tungsten sputtered from the divertor target plates will get mixed with the relatively dense divertor plasma $\left(n=10^{14-15} \mathrm{~cm}^{-3}\right)$, and from there, the ions could be transported to the edge and core plasmas. Concentration measurements of tungsten impurity ions in the divertor volume are thus critical for assessing tungsten flux rates. Based on the tungsten ionization energies [3], it can be expected that, neglecting transport, approximately the first ten charge states will have high abundances. For divertor plasmas, it is thus the atomic data for few-times ionized tungsten that are needed.

The first four tungsten spectra have been extensively studied; see, e.g., the atomic data compilations by Kramida and Shirai [4,5] and Kramida [6]. The first two spectra are mainly in the visible and ultraviolet range, whereas W III and W IV mostly fall in the vacuum ultraviolet (VUV) interval. With increasing ionization degree, the emission shifts toward shorter wavelengths and Yb-like W v is the first spectrum with experimentally known lines in the extreme ultraviolet (EUV) region from triggered- and sliding-spark measurements by Churilov et al. [7] and Kildiyarova et al. [8]. Meijer [9] and later Kaufman and Sugar [10,11] measured VUV and EUV lines from Tm-like W VI using sliding-spark discharges. Recently, photoionization experiments were carried out on tungsten charge states up to five-times ionized at the ALS synchrotron by Müller et al. [12]. Sugar and Kaufman measured VUV and EUV lines from the closed-shell Er-like $\mathrm{W}^{6+}$ ion using a sliding-spark light source [13]. The W VII spectrum was further investigated by Wyart et al., who identified a number of lines in the EUV [14]. Recent spark measurements by Ryabtsev et al. have allowed a large number of lines in W VIII to be identified [15,16], resulting in a detailed analysis of the atomic structure of Ho-like $\mathrm{W}^{7+}$. These measurements have provided very accurate line positions, but due to the high densities of the spark light sources, the spectral features will likely not resemble the emission from divertor plasmas. Emission from low charge states of tungsten has been observed in magnetic fusion plasmas, however, not with enough resolution to allow for detailed spectroscopic measurements. The Ho-like W VIII spectrum was observed by Veres et al. in the MT-1M tokamak [17], and Clementson et al. recorded spectra of Tm-like through Ho-like W VI-VIII at the SSPX spheromak [18]. However, without additional laboratory or theoretical reference data, such plasma spectroscopic measurements are difficult to interpret.

Detailed laboratory measurements on tungsten ions are well suited for electron beam ion traps (EBITs), where charge-state selection is good and the ion temperature and electron density low. Several studies of tungsten in various ionization stages and spectral ranges have been conducted using EBITs; see, e.g., [19-33]. Although designed to have a very wide electron-beam energy range, EBITs have mostly been used at energies above several hundred electron volts, leading to a focus on highly or moderately charged ions with little attention on the first few charge states.

In this work, the low-energy capabilities of the electron beam ion trap facility at the Lawrence Livermore National Laboratory [34] have been utilized to investigate the EUV emission from few-times ionized tungsten. The present measurements were performed using EBIT-I, which is the original electron beam ion trap developed at Livermore thirty years ago [35-37], by scanning the beam energy in the range of 30-300 eV and exciting the tungsten spectra expected from tokamak divertor plasmas. 


\section{EBIT Spectroscopy}

The spectra of the few-times ionized tungsten were experimentally investigated using the EBIT-I electron beam ion trap during two run campaigns. In the first, a survey study was conducted to see how the EUV spectra evolve as a function of collision energy. The second study focused on high-resolution measurements at low excitation energies. The experimental setup and run conditions were similar for the two campaigns; tungsten was supplied to a 40-V trap by sublimation of tungsten hexacarbonyl, $\mathrm{W}(\mathrm{CO})_{6}$, which diffused from a vial radially into the 1-mA electron beam. By varying the voltage of the drift-tube assembly (the same as the middle drift-tube voltage) while keeping the voltage of the electron gun fixed, the electron-ion collision energy was scanned in the 30-300 eV interval with an energy spread of a few tens of electron volts.

The wavelength calibrations were done by measuring well-known reference lines from oxygen spectra before, between and after tungsten data acquisitions. Carbon dioxide, $\mathrm{CO}_{2}$, gas was introduced into the electron beam using a ballistic gas injector mounted on one of the radial ports surrounding the trap. When the $\mathrm{CO}_{2}$ molecules collide with 2-keV beam electrons, they dissociate, and subsequently, the constituents become ionized and trapped in a $100-\mathrm{V}$ potential well. The strong emission from O IV-V provided line positions to which polynomials could be fitted. Reference wavelength values were found in the Kelly line lists [38] and originate from the works of Bromander [39], Bockasten and Johansson [40] and Edlén [41].

\subsection{Survey Measurements}

The spectra were studied using a moderate-resolution broad-band grating spectrometer of the same type used for grazing-incidence measurements at the SSPX, NSTX, LTX and Alcator C-Mod magnetic fusion facilities [42-47]. The instrument employs a 1200-line/mm flat-field grating with a radius of curvature of $5.6 \mathrm{~m}$. The diffracted rays are focused on a Princeton Instruments back-illuminated CCD camera with a $1024 \times 1340$ array of pixels, each of a size of $20 \mu \mathrm{m} \times 20 \mu \mathrm{m}$. The data were recorded during one or several thirty-minute acquisitions. The images were then added together and filtered for cosmic rays before being analyzed.

Wavelength scales were established by fitting second-degree polynomials to oxygen lines in the 150-300 A interval. Many of these lines were not fully resolved, and the intensity-weighted centroid positions were used, with intensity values taken from the Kelly line lists [38], resulting in line-position accuracies better than $0.4 \AA$. Because of the large detector, it is not possible to have the entire image in good focus. The spectral resolution therefore varies slightly over the images with typical line widths of $0.4 \AA$ in the central parts increasing to $0.8 \AA$ toward the edges.

The electron-beam energy was varied in the range from 31 up to $293 \mathrm{eV}$, which should cover many of the low tungsten charge states [3]. Figures 1 and 2 show observed spectra at ten beam energies. 


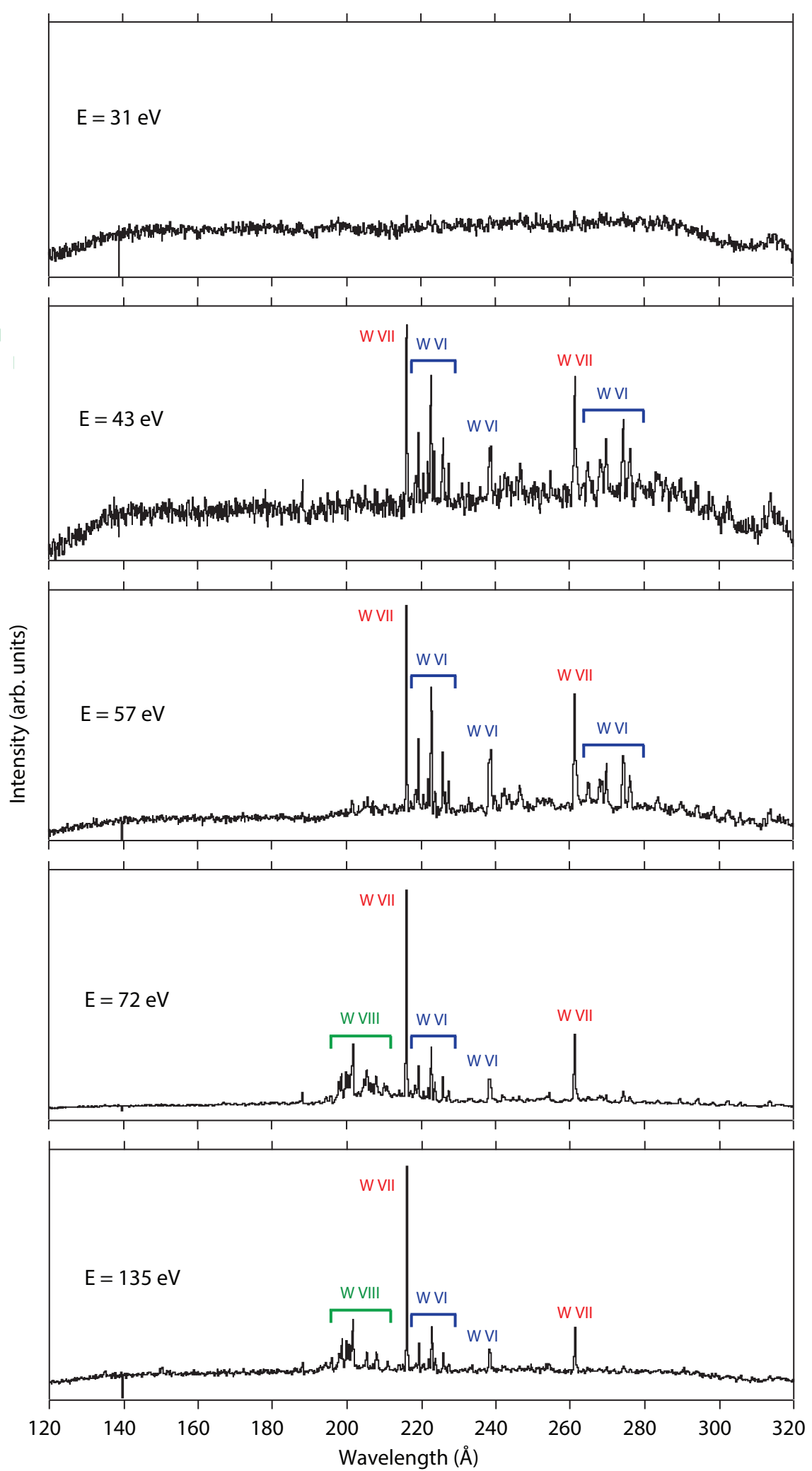

Figure 1. Tungsten spectra measured at EBIT-I at nominal electron-beam energies of $\mathrm{E}=31-135 \mathrm{eV}$. Strong lines and intervals are labeled according to dominating spectra. 


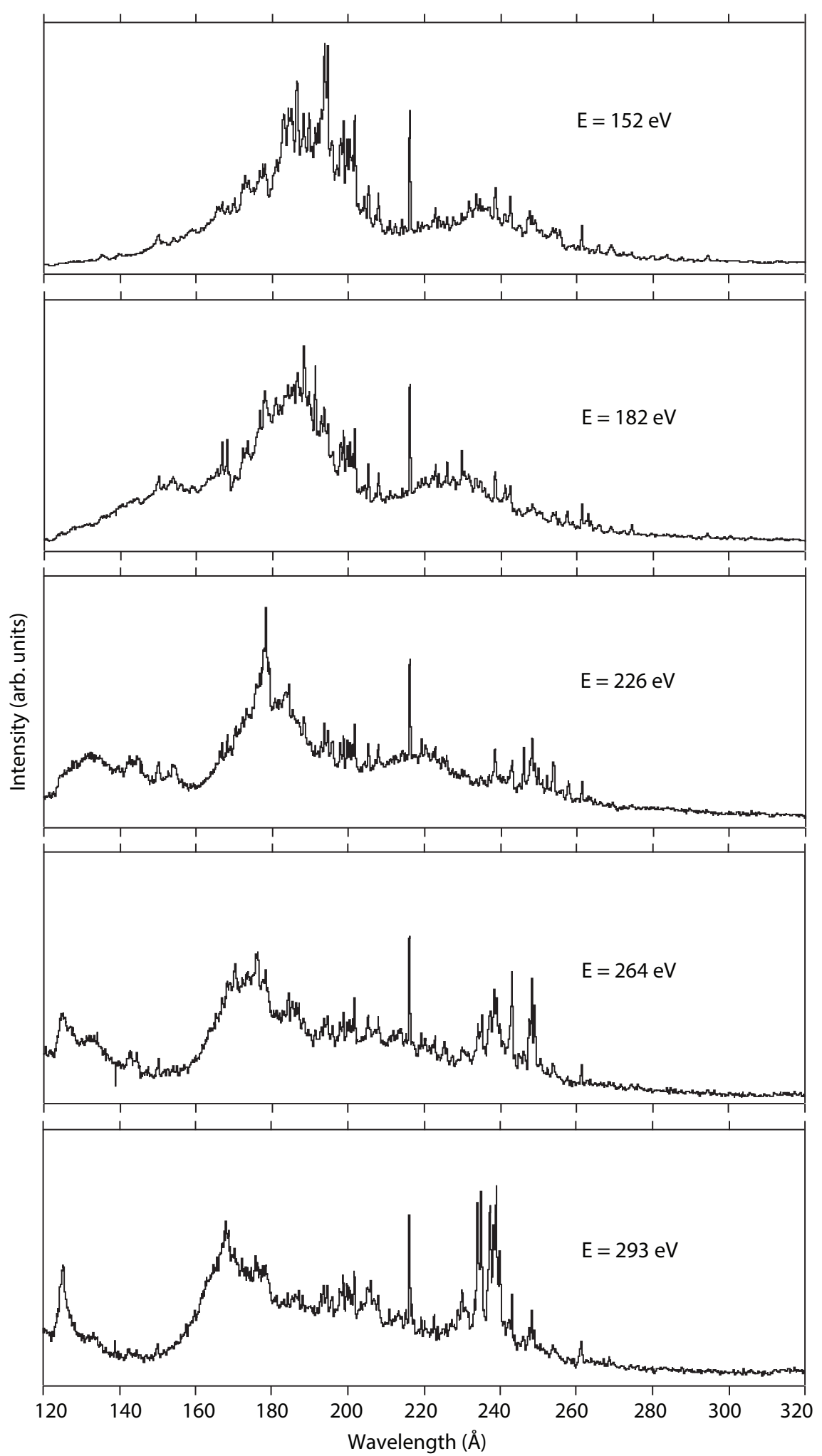

Figure 2. Tungsten spectra measured at EBIT-I at nominal electron-beam energies of $\mathrm{E}=152-293 \mathrm{eV}$. 


\subsection{High-Resolution Measurements}

The high-resolution measurements were done using a grazing-incidence flat-field grating spectrometer [48]. The spectrometer, which employs a varied-spaced grating with an average ruling of 2400 lines $/ \mathrm{mm}$ and a radius of a curvature of $44.3 \mathrm{~m}$, was originally designed for EBIT measurements in the 10-50 A wavelength range, but the good performance of the instrument motivated an upgrade, and the diffraction-angle coverage has since been extended to measure wavelengths up to around $250 \AA$. Recently, a similar instrument has been developed with an even larger wavelength coverage from below 10 to above $300 \AA$ [49]. For these measurements, the spectrometer made use of a Princeton Instruments CCD camera of the same kind as used for the survey spectrometer. Tungsten emission was measured at two exit-arm positions, one covering the wavelength interval 201.5-223.8 $\AA$ and the other 187.6-209.0 ̊. Data were collected at several beam energies for multiple thirty-minute exposures. For each excitation energy, the data were added and filtered for cosmic rays. The images were also rotated to correct for a small tilt of the detector relative to the grating.

For the long-wavelength spectrometer position, tungsten emission was studied at electron-beam energies $E=52,74,103$ and $127 \mathrm{eV}$. The tungsten spectra at 52 and $74 \mathrm{eV}$ show lines between 216 and $223 \AA$. In the $103-\mathrm{eV}$ spectrum, a few weak lines appear in the shorter wavelength range, and in the 127-eV spectrum, all lines are shown clearly; this spectrum was therefore selected for line analysis; see Figure 3. The wavelength scale was established by fitting intensity-weighted second-degree polynomials using the $\mathrm{O}$ IV line at 207.239 $\AA$ [38,39] and the O v lines at 215.245 and 220.352 $\mathrm{A}$ [38,40] from $\mathrm{CO}_{2}$ measurements before and after the tungsten data acquisition. The quality of the wavelength versus detector-position function was then tested on the O IV line at $207.183 \AA[38,39]$ and the O V line at $222.235 \AA[38,40]$. The statistical uncertainty of the tested line positions was better than $4 \mathrm{~m} \AA$. Nevertheless, the oxygen calibration spectrum after the tungsten data acquisitions was found to have a large drift (around $10 \mathrm{~m} \AA$ ) relative the oxygen spectrum before the tungsten data collection. This drift is assumed to be due to the thermal motions of the EBIT-spectrometer setup and limits the measurement accuracy. To lessen the position uncertainty for the majority of the observed lines, the previously known Er-like $\mathrm{W}^{6+}$ line from the $\left(5 p^{6}\right)_{0}-\left(5 p_{1 / 2} 5 p_{3 / 2}^{4} 5 d_{3 / 2}\right)_{1}$ transition at 216.219(5) $\AA$, as measured by Sugar and Kaufman [13], was used as a reference line to which the wavelength scale of the first oxygen calibration spectrum was anchored by shifting it $18 \mathrm{~m} \AA$. Comparing the first oxygen spectrum to the second, there is a relative difference between them of nearly $2 \mathrm{~m} \AA$ between the edges of the detector. Considering that it is possible that the stretch increases with shift and noting that the shift between the two oxygen spectra is about half of the shift of the oxygen relative to the tungsten spectrum, the dispersion is assumed accurate to around $4 \mathrm{~m} \AA$. Wavelengths for all lines between 206 and $223 \AA$ with peak intensities above 250 counts were determined; see Table 1. Each line position was fitted with a Gaussian profile. The resulting full width at half maximum (FWHM) of around $30 \mathrm{~m} \AA$ and the number of counts were used to estimate the counting-statistical uncertainty, which, for most lines, was no more than $1 \mathrm{m \AA}$. The total uncertainty for the measured line positions is estimated to be better than $10 \mathrm{~m} \AA$.

For the 187.6-209.0 $\AA$ spectrometer position, tungsten data were acquired at electron-beam energies $E=75,99$ and $128 \mathrm{eV}$. The 75-eV spectrum did not show any lines, whereas the 99- and 128-eV spectra showed plenty of strong lines. The 128-eV spectrum was chosen for analysis; see Figure 4. 
The wavelength scale was calculated by fitting second-degree polynomials of the $\mathrm{O}$ IV lines at 196.009 and $207.239 \AA$ [38,39] and the $\mathrm{O}$ V line at $192.906 \AA[38,41]$ for $\mathrm{CO}_{2}$ spectra acquired before and after the tungsten measurement. The dispersion from the pre-tungsten oxygen spectrum was applied to the tungsten spectrum and anchored to the position of the line from the Er-like $\mathrm{W}^{6+}\left(5 p^{6}\right)_{0}-\left(5 p_{1 / 2} 5 p_{3 / 2}^{4} 6 s_{1 / 2}\right)_{1}$ transition at 188.159(5) $\AA$, which has been measured by Sugar and Kaufman [13], by shifting the scale $24 \mathrm{~m} \AA$. The uncertainty in the dispersion between the two calibration spectra is $15 \mathrm{~m} \AA$, as judged from the difference in wavelength from edge to edge of the image. It is noted, though, that two of the lines at the right-hand side of the tungsten spectrum are also found in the long-wavelength tungsten spectrum with centroid positions agreeing within $5 \mathrm{~m} \AA$. Lines with peak intensities above 250 counts were fitted to Gaussian profiles. The line widths were here around $33 \mathrm{~m} \AA$, resulting in statistical uncertainties of $1 \mathrm{~m} \AA$ for all lines. The total line-position uncertainty is thus estimated to be around $15 \mathrm{~m} \AA$. The measured wavelengths are listed in Table 1 .

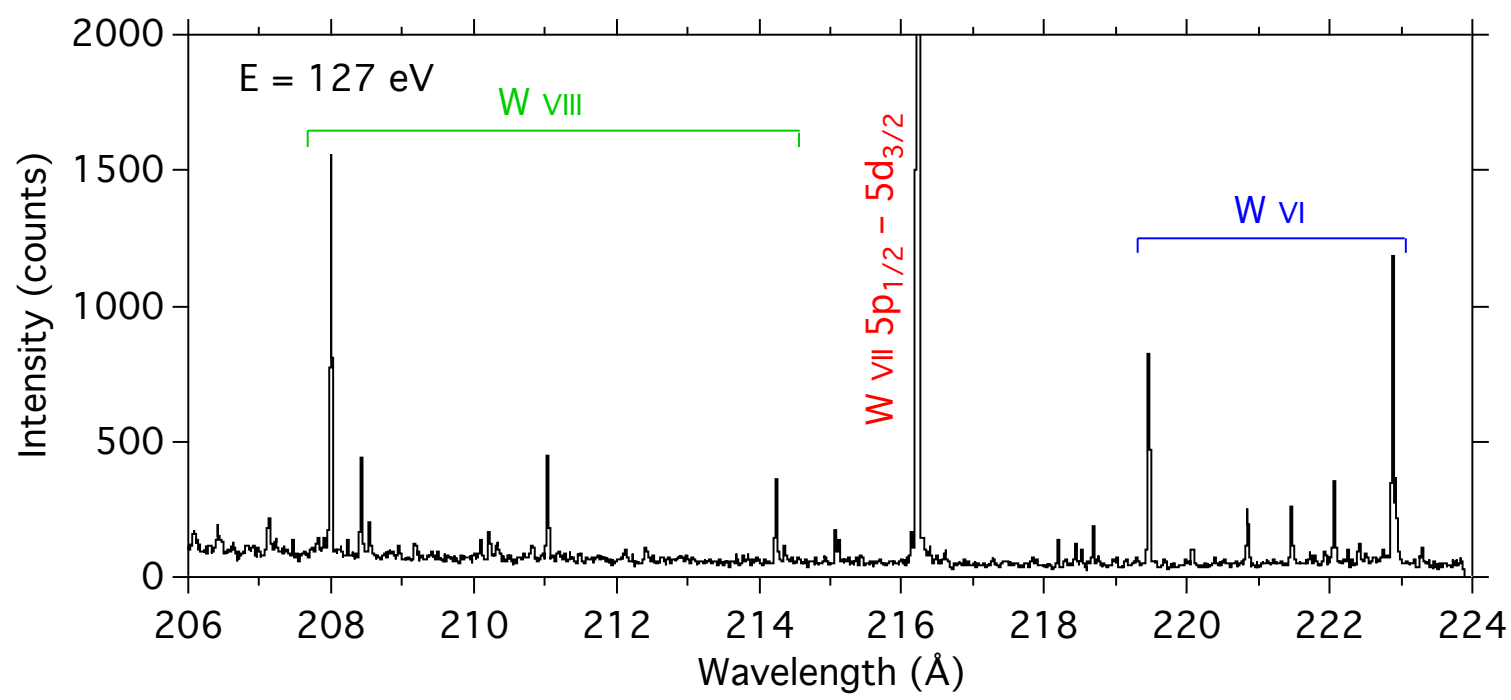

Figure 3. Measured spectra of tungsten at EBIT-I at a nominal beam energy of $E=127 \mathrm{eV}$.

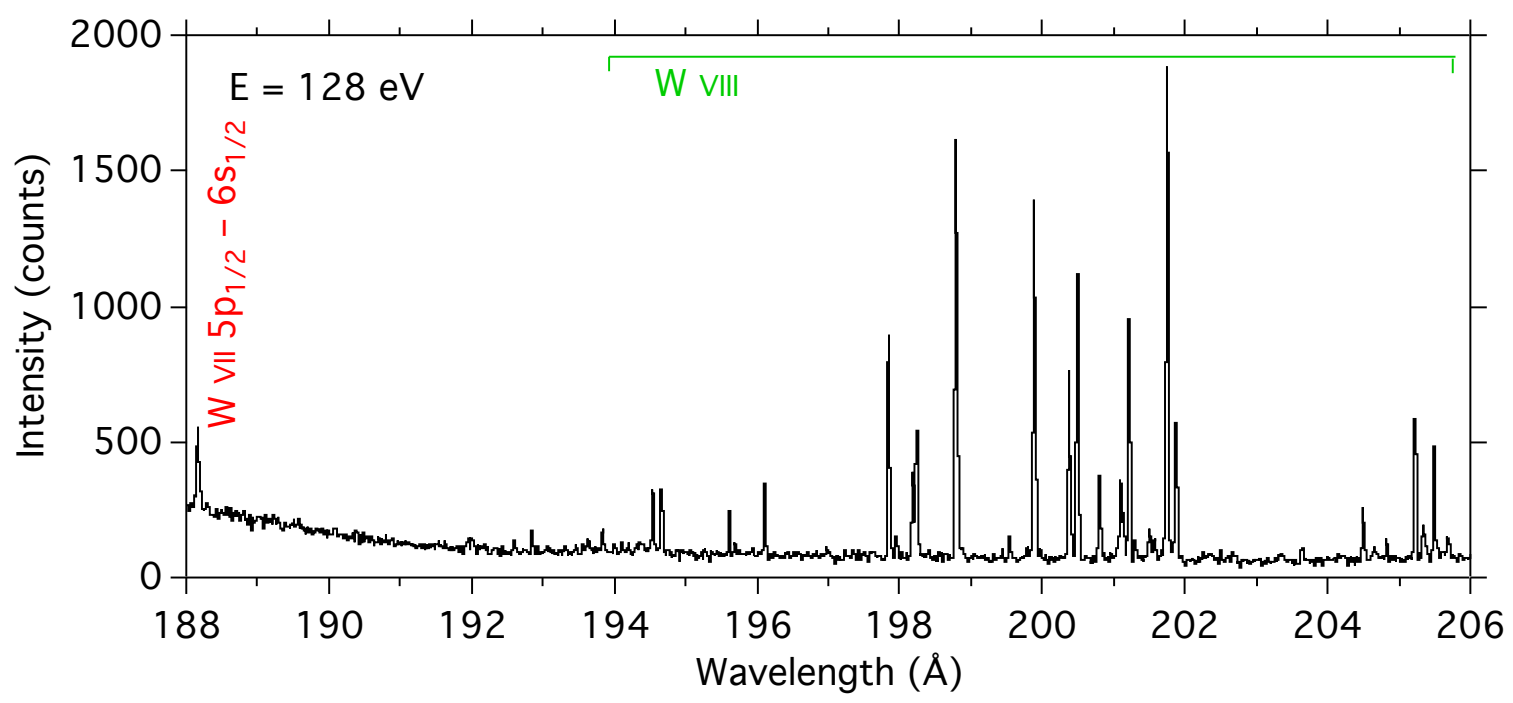

Figure 4. Measured spectra of tungsten at EBIT-I at a nominal beam energy of $E=128 \mathrm{eV}$. 
Table 1. Measured line positions in the Tm-like W VI and Ho-like W VIII spectra. $\lambda_{\text {exp }}$ are experimental wavelengths from this work and $\lambda_{\text {prev }}$ previously measured line positions in W VIII by Ryabtsev et al. [16].

\begin{tabular}{|c|c|c|c|c|}
\hline Sequence & $\lambda_{e x p}(\AA)$ & $\lambda_{\text {prev }}(\AA)$ [16] & Lower level [16] & Upper level [16] \\
\hline \multirow[t]{22}{*}{ Ho } & $194.530(15)$ & $194.527(5)$ & $\left(4 f^{13} 5 p^{6}\right)_{7 / 2}$ & $\left(4 f^{13} 5 p^{5} 6 s\right)_{5 / 2}$ \\
\hline & $194.653(15)$ & & & \\
\hline & $196.096(15)$ & 196.093(5) & $\left(4 f^{13} 5 p^{6}\right)_{5 / 2}$ & $\left(4 f^{13} 5 p^{5} 6 s\right)_{7 / 2}$ \\
\hline & $197.838(15)$ & $197.835(5)$ & $\left(4 f^{13} 5 p^{6}\right)_{5 / 2}$ & $\left(4 f^{12} 5 p^{6} 5 d\right)_{3 / 2}$ \\
\hline & $198.176(15)$ & $198.171(5)$ & $\left(4 f^{13} 5 p^{6}\right)_{7 / 2}$ & $\left(4 f^{13} 5 p^{5} 6 s\right)_{9 / 2}$ \\
\hline & $198.233(15)$ & $198.229(5)$ & $\left(4 f^{13} 5 p^{6}\right)_{5 / 2}$ & $\left(4 f^{13} 5 p^{5} 6 s\right)_{5 / 2}$ \\
\hline & $198.782(15)$ & $198.779(5)$ & $\left(4 f^{13} 5 p^{6}\right)_{7 / 2}$ & $\left(4 f^{13} 5 p^{5} 6 s\right)_{7 / 2}$ \\
\hline & $199.881(15)$ & $199.875(5)$ & $\left(4 f^{13} 5 p^{6}\right)_{7 / 2}$ & $\left(4 f^{13} 5 p^{5} 6 s\right)_{5 / 2}$ \\
\hline & $200.371(15)$ & $200.367(5)$ & $\left(4 f^{13} 5 p^{6}\right)_{5 / 2}$ & $\left(4 f^{13} 5 p^{5} 5 d\right)_{5 / 2}$ \\
\hline & $200.487(15)$ & $200.483(5)$ & $\left(4 f^{13} 5 p^{6}\right)_{7 / 2}$ & $\left(4 f^{13} 5 p^{5} 5 d\right)_{7 / 2}$ \\
\hline & $200.793(15)$ & $200.787(5)$ & $\left(4 f^{13} 5 p^{6}\right)_{7 / 2}$ & $\left(4 f^{13} 5 p^{5} 5 d\right)_{5 / 2}$ \\
\hline & $201.085(15)$ & $201.079(5)$ & $\left(4 f^{14} 5 p^{5}\right)_{3 / 2}$ & $\left(4 f^{14} 5 p^{4} 5 d\right)_{3 / 2}$ \\
\hline & $201.209(15)$ & $201.205(5)$ & $\left(4 f^{13} 5 p^{6}\right)_{5 / 2}$ & $\left(4 f^{13} 5 p^{5} 6 s\right)_{7 / 2}$ \\
\hline & $201.743(15)$ & $201.739(5)$ & $\left(4 f^{13} 5 p^{6}\right)_{7 / 2}$ & $\left(4 f^{13} 5 p^{5} 5 d\right)_{9 / 2}$ \\
\hline & $201.869(15)$ & $201.864(5)$ & $\left(4 f^{13} 5 p^{6}\right)_{5 / 2}$ & $\left(4 f^{13} 5 p^{5} 5 d\right)_{7 / 2}$ \\
\hline & $204.490(15)$ & & & \\
\hline & 205.221(15) & 205.221(5) & $\left(4 f^{13} 5 p^{6}\right)_{5 / 2}$ & $\left(4 f^{12} 5 p^{6} 5 d\right)_{3 / 2}$ \\
\hline & $205.480(15)$ & $205.479(5)$ & $\left(4 f^{14} 5 p^{5}\right)_{3 / 2}$ & $\left(4 f^{14} 5 p^{4} 6 s\right)_{3 / 2}$ \\
\hline & 207.994(10) & & & \\
\hline & $208.417(10)$ & $208.420(5)$ & $\left(4 f^{14} 5 p^{5}\right)_{3 / 2}$ & $\left(4 f^{14} 5 p^{4} 5 d\right)_{5 / 2}$ \\
\hline & 211.031(10) & $211.027(5)$ & $\left(4 f^{14} 5 p^{5}\right)_{3 / 2}$ & $\left(4 f^{14} 5 p^{4} 5 d\right)_{3 / 2}$ \\
\hline & $214.235(10)$ & $214.229(5)$ & $\left(4 f^{14} 5 p^{5}\right)_{3 / 2}$ & $\left(4 f^{12} 5 p^{6} 5 d\right)_{5 / 2}$ \\
\hline \multirow[t]{5}{*}{$\mathrm{Tm}$} & 219.461(10) & & & \\
\hline & $220.839(10)$ & & & \\
\hline & $221.454(10)$ & & & \\
\hline & $222.053(10)$ & & & \\
\hline & $222.879(10)$ & & & \\
\hline
\end{tabular}

\section{Spectral Analysis}

The character of the measured survey spectra is very different for excitation energies below and above $150 \mathrm{eV}$; see Figures 1 and 2. At lower energies, the emission consists of isolated spectral lines, whereas at higher energies, the spectra are made up of emission bands with only a smaller number of identifiable lines. The analysis is therefore focused on the low-energy emission, which, based on previous spectral modeling calculations [18], is believed to originate from Yb-like W v, Tm-like W VI, Er-like W VII and Ho-like W VIII. The evolution of emission with electron-beam energy together with ab initio wavelength calculations was utilized to interpret the low-energy survey and high-resolution spectra. 


\subsection{Theoretical Atomic Data}

To support the analysis of the experimental data, the measured spectra were compared to calculations of $\mathrm{W}^{5+}, \mathrm{W}^{6+}$ and $\mathrm{W}^{7+}$ by Ulyana I. Safronova [50,51] using a modified version of the Cowan code [52]. EUV transitions connecting to the ground-configuration levels for these spectra are presented in Figure 5, where the weighted radiative transition probability rates as a function of wavelength are shown for the three spectra W VI-VIII.

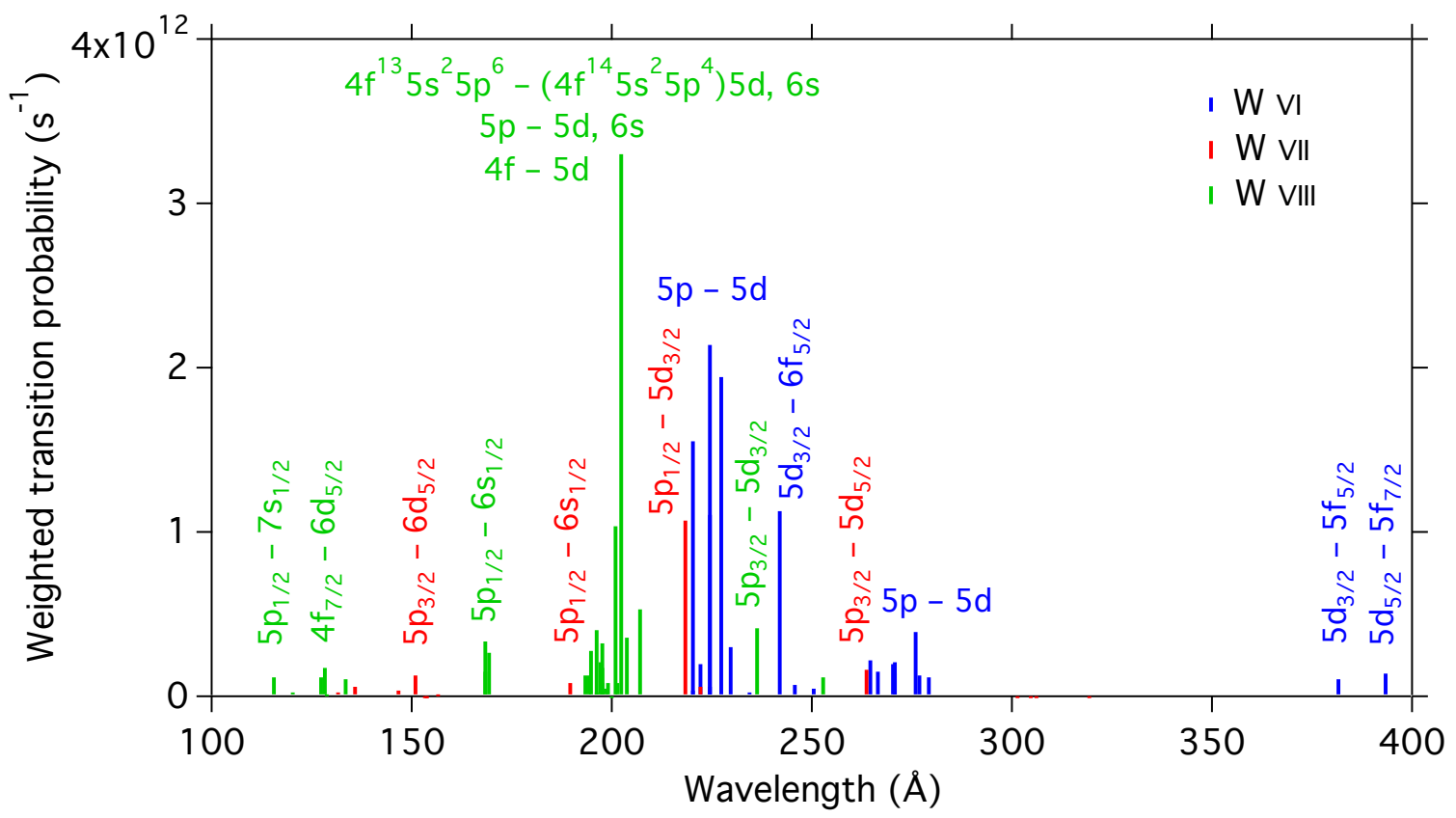

Figure 5. Wavelengths and weighted radiative transition probability rates for transitions to the ground configurations in W VI-VIII. Cowan calculations courtesy of U.I. Safronova.

All transitions considered in the $\mathrm{Tm}$-like $\mathrm{W}^{5+}$ ion connect to the $4 f^{14} 5 s^{2} 5 p^{6} 5 d_{3 / 2}$ ground level or the $5 d_{5 / 2}$ level. Three of the transitions involve the electron outside the filled $5 p^{6}$ subshell, whereas the others are inner-shell transitions from $5 p^{-1} 5 d^{2}$ excited levels. Most of the $5 p-5 d$ transitions fall in either of two groups; between 220 and $230 \AA$ or between 260 and $280 \AA$. The quality of the calculated W VI line positions may be judged by comparing them to experimentally known EUV lines. The $5 d_{3 / 2}-5 f_{5 / 2}$ and $5 d_{5 / 2}-5 f_{7 / 2}$ transitions have been measured by Sugar and Kaufman at 382.145(5) and 394.133(5) A, respectively [11]. The theoretical wavelengths are near the experimental values with 381.786 and $393.479 \AA$.

The calculated transitions in the Er-like $\mathrm{W}^{6+}$ ion connect to the $\left(4 f^{14} 5 s^{2} 5 p^{6}\right)_{0}$ ground level. All of the calculated W VII lines may be compared to the line positions measured by Sugar and Kaufman [13]. Most lines are within three ångströms, such as the $5 p^{6}-5 p 5 d$ transitions measured at 216.219 and 261.387(5) $\AA$ and calculated at 218.516(5) and 263.683(5) $\AA$ and the transition from $(5 p 6 s)_{1}$ measured at $188.159(5) \AA$ and calculated at $189.716 \AA$. The theoretical line positions around $300 \AA$, however, are not as good and are off by more than $10 \AA$.

All but one of the calculated transitions in the Ho-like $\mathrm{W}^{7+}$ ion connect to the $\left(4 f^{-1} 5 s^{2} 5 p^{6}\right)_{5 / 2,7 / 2}$ ground-configuration levels. Although Ryabtsev et al. $[15,16]$ have measured a large number of lines 
in the W VIII spectrum, it is difficult to evaluate the quality of the theoretical wavelengths, because the transition assignments are not the same. The labeled transitions in Figure 5 are from the present Cowan calculations.

While most of the calculated wavelengths appear to be within a few ångströms of actual values, some transitions are off by more. This accuracy is not enough for unique identifications of unknown lines, but the calculations point out spectral intervals where strong transitions from W VI-VIII are expected.

\subsection{Analysis}

A comparison of the calculated transition probabilities and wavelengths of Figure 5 with the measured low-energy spectra of Figure 1 supports the notion that the emission mainly consists of W VI-VIII with possibly a few lines belonging to the $\mathrm{W} v$ spectrum. Based on the theoretical data, lines and intervals where a certain spectrum dominates are indicated in the survey spectra of Figure 1. The top panel shows the data recorded at $E=31 \mathrm{eV}$, but no lines can be seen here. However, already at the next nominal beam energy of $43 \mathrm{eV}$, there are many lines appearing, most of them likely belonging to Yb-like $\mathrm{W} v$ and Tm-like W VI with a few known Er-like W VII lines also showing [13]. The emission essentially looks the same at $E=57 \mathrm{eV}$, but in the forth panel, at $72 \mathrm{eV}$, new lines appear around $200 \AA$, interpreted as the entry of Ho-like W VIII. The same lines are found in the bottom panel of Figure 1 showing the data at $E=$ $135 \mathrm{eV}$. For excitation energies above $150 \mathrm{eV}$, shown in Figure 2, the EUV emission is very different, with many strong densely spaced lines. As the excitation energy varies from $152 \mathrm{eV}$ in the top panel to $293 \mathrm{eV}$ in the bottom panel, the evolution of emission bands may be followed, but individual features associated with specific charge states are difficult to identify at this resolution. Although having an uncertainty of a few tens of electron volts in the EBIT excitation energy, the ionization energy of $141.2 \mathrm{eV}$ for Ho-like $\mathrm{W}^{7+}$ [3] suggests that the complex tungsten emission starts with the Dy-like W IX spectrum.

The observed lines in the high-resolution data were assigned to tungsten spectra based on the EUV emission evolution with beam energy and the calculated atomic data. In the long-wavelength spectrum (see Figure 3), the five measured lines between 219 and $223 \AA$, which have not previously been reported, are identified as Tm-like W VI and are likely from $5 p-5 d$ transitions. The lines on the left-hand side of the strong W VII $\left(5 p^{6}\right)_{0}-\left(5 p_{1 / 2} 5 p_{3 / 2}^{4} 5 d_{3 / 2}\right)_{1}$ reference line at 216.219(5) $\AA$ [13] are identified as Ho-like W VIII. For three of the four measured lines, these identifications are corroborated by the work of Ryabtsev et al. [16]. Aside from the Er-like W VII $\left(5 p^{6}\right)_{0}-\left(5 p_{1 / 2} 5 p_{3 / 2}^{4} 6 s_{1 / 2}\right)_{1}$ reference line at 188.159(5) $\AA$ [13], all lines in the short-wavelength spectrum are assigned to Ho-like W VIII; see Figure 4. Most of these lines have been measured by Ryabtsev et al. [16]. The measured wavelengths from Tm-like W VI and Ho-like W VIII are listed in Table 1.

\section{Results and Discussion}

The broad-band spectra of Figures 1 and 2 display how the EUV emission signature of few-times ionized tungsten change with excitation energy in an interval relevant for tokamak divertor plasmas. In the simple low-energy spectra, several of the W VII lines measured by Sugar and Kaufman [13] may be seen, although most of them are fairly weak. Due to its closed-shell structure, Er-like $\mathrm{W}^{6+}$ is likely abundant over a wide temperature range, and in fact, the strongest two spectral lines are seen in all 
but the first of the panels of Figures 1 and 2. These two lines, at 216.219(5) and 261.387(5) $\AA$, are from the $\left(5 p^{6}\right)_{0}-\left(5 p_{1 / 2} 5 p_{3 / 2}^{4} 5 d_{3 / 2}\right)_{1}$ and $\left(5 p^{6}\right)_{0}-\left(5 p_{1 / 2}^{2} 5 p_{3 / 2}^{3} 5 d_{5 / 2}\right)_{1}$ transitions, respectively [13]. At the low energies, the two lines have an excitation-energy-dependent intensity ratio. If this trend is similar in the emission from a Maxwellian plasma, the relative line intensities may then be used for local electron-temperature measurements in a divertor volume. It is, however, also possible that this line ratio is sensitive to electron density, since the $261.387 \AA$ line was noted to be significantly stronger than the 216.219 A line in the spectra observed from the spark discharges by Sugar and Kaufman, whereas in the spectra from the low-density SSPX spheromak plasmas [18] and in the present EBIT data, the reversed ratio is seen. The low-energy spectra show several lines belonging to Tm-like W VI. The $5 d_{3 / 2}-5 f_{5 / 2}$ transition at 382.145(5) $\AA$ [11] has previously been observed in the SSPX spheromak [18]. Here, the next transition in the Rydberg series, $5 d_{3 / 2}-6 f_{5 / 2}$, is found at $238.6 \AA$ as a relatively strong broad line. The other observed Tm-like W VI lines are, based on the Cowan calculations, $5 p-5 d$ transitions and are located within the two intervals between 220 and $230 \AA$ and 260 and $280 \AA$. Ho-like W VIII have many closely spaced lines appearing between 195 and $215 \AA$ from transitions connecting ground-configuration levels, as seen in the low-energy survey spectra and the theoretical spectrum of Figure 5.

Applying the high-resolution spectrometer on the region near $200 \AA$, the wavelengths of five W VI lines and twenty-two W VIII lines could be determined with line-position accuracies of 10-15 m see Table 1 and Figures 3 and 4. The five W VI lines are likely $5 p-5 d$ transitions and have not previously been reported. Nineteen of the W VIII lines have been measured with $5 \mathrm{~m} \AA$ accuracy by Ryabtsev et al. [16]. The line positions of the two measurements are within $6 \mathrm{~m} \AA$ from each other and, thus, provide a good consistency check of the wavelength values. The two Er-like W VII lines at 216.219(5) and 188.159(5) $\AA$ that were observed in the high-resolution spectra and used as reference wavelengths are broader than the other measured lines with FWHM of 46 and $39 \mathrm{~m} \AA$, respectively. This should be compared to 27-34 $\mathrm{m} \AA$ for the other lines in Figure 3 and 28-37 $\mathrm{m} \AA$ for the lines in Figure 4 . The $188.159 \AA$ line, from the $\left(5 p^{6}\right)_{0}-\left(5 p_{1 / 2} 5 p_{3 / 2}^{4} 6 s_{1 / 2}\right)_{1}$ transition, is observed close to the edge of the detector, and the wideness of the line may be due to having a worse focus here; however, the $216.219 \AA$ line, from the $\left(5 p^{6}\right)_{0}-\left(5 p_{1 / 2} 5 p_{3 / 2}^{4} 5 d_{3 / 2}\right)_{1}$ transition, is in the middle of the detector with well-resolved lines on both sides of it. It is interesting that the two observed W VII lines are wider than the other measured lines. The reason for this is unclear.

The relatively simple EUV emission of W V-VIII expected from low-temperature tokamak divertor plasmas should be suitable for spectroscopic diagnostics focusing on isolated lines. Such lines may be employed for measuring concentrations of tungsten ions and, thus, also transport from the wall to the plasma. In the ITER tokamak tungsten influx rates are planned to be assessed using a strong line at $4008.7506 \AA$ in W I [53]. There are, however, concerns that concentration measurements of neutral tungsten near the wall may be misleading as a measure of tungsten influx rates, since the sputtered tungsten atoms have a high probability of getting redeposited on the target surface $[53,54]$. If, additionally, also spectral lines from the first few charge states could be monitored, a more accurate picture of the amount of tungsten entering the plasma may be achieved. Erosion and poloidal migration of tungsten in the Alcator C-Mod divertor have recently been studied by Barnard et al. [55]. Farther from the wall where the temperature is high enough to have a significant population of tungsten ions in charge states above Ho-like $\mathrm{W}^{7+}$, the complex EUV emission might make spectroscopic investigations 
on isolated lines difficult. This emission, however, will be very important for the divertor power balance. Knowledge of the radiation profile in the divertor is useful for determining how much external impurity seeding is needed to provide the necessary radiative cooling.

\section{Acknowledgments}

This work was performed under the auspices of the United States Department of Energy by Lawrence Livermore National Laboratory under Contract No. DE-AC52-07NA-27344. The work was carried out as part of the Livermore WOLFRAM Project and the International Atomic Energy Agency (IAEA) Coordinated Research Project Spectroscopic and Collisional Data for Tungsten from $1 \mathrm{eV}$ to $20 \mathrm{keV}$. The authors wish to thank Ulyana I. Safronova for providing the Cowan calculations and Edward W. Magee and David A. Layne for technical support.

\section{Author Contributions}

Joel Clementson and Thomas Lennartsson measured and analyzed spectra. Peter Beiersdorfer was involved in discussions and in leading the scientific efforts at the Livermore EBIT facility.

\section{Conflicts of Interest}

The authors declare no conflict of interest.

\section{References}

1. Clementson, J.; Beiersdorfer, P.; Lennartsson, T. Atomic data of tungsten for current and future uses in fusion and plasma science. AIP Conf. Proc. 2013, 1525, 78-83.

2. Beiersdorfer, P.; May, M.J.; Scofield, J.H.; Hansen, S.B. Atomic physics and ionization balance of high- $Z$ ions: Critical ingredients for characterizing and understanding high-temperature plasmas. High Energy Density Phys. 2012, 8, 271-283.

3. Kramida, A.E.; Reader, J. Ionization energies of tungsten ions: $\mathrm{W}^{2+}$ through $\mathrm{W}^{71+}$. At. Data Nucl. Data Tables 2006, 92, 457-479.

4. Kramida, A.E.; Shirai, T. Compilation of wavelengths, energy levels, and transition probabilities for W I and W II. J. Phys. Chem. Ref. Data 2006, 35, 423-683.

5. Kramida, A.E.; Shirai, T. Energy levels and spectral lines of tungsten, W III through W LXXIV. At. Data Nucl. Data Tables 2009, 95, 305-474.

6. Kramida, A. Recent progress in spectroscopy of tungsten. Can. J. Phys. 2011, 89, 551-570.

7. Churilov, S.S.; Kildiyarova, R.R.; Joshi, Y.N. Analyses of the $5 d^{2}-5 d 7 p$ transitions in the Ta IV and W v spectra. Can. J. Phys. 1996, 74, 145-149.

8. Kildiyarova, R.R.; Churilov, S.S.; Joshi, Y.N. Analysis of the $5 d 5 f$ configuration of trebly ionized tantalum and quadruply ionized tungsten: Ta IV and W V. Phys. Scr. 1996, 53, 454-460.

9. Meijer, F.G. The sixth spectrum of tungsten, W VI. Physica 1974, 73, 415-420.

10. Kaufman, V.; Sugar, J. Wavelengths, classifications, and ionization energies in the isoelectronic sequences from Yb II and Yb III through Bi XV and Bi XVI. J. Opt. Soc. Am. 1976, 66, 1019-1025. 
11. Sugar, J.; Kaufman, V. Identification of $5 g$ and $6 g$ terms and revised ionization energies in the Yb II $4 f^{14} n l$ isoelectronic sequence. J. Opt. Soc. Am. 1979, 69, 141-143.

12. Müller, A.; Schippers, S.; Kilcoyne, A.L.D.; Esteves, D. Photoionization of tungsten ions with synchrotron radiation. Phys. Scr. 2011, 2011, 014052.

13. Sugar, J.; Kaufman, V. Seventh spectrum of tungsten (W VII); resonance lines of Hf V. Phys. Rev. A 1975, 12, 994-1012.

14. Wyart, J.F.; Kaufman, V.; Sugar, J. The $4 f^{13} 5 f$ configuration in the isoelectronic sequence of Yb III. Phys. Scr. 1981, 23, 1069-1078.

15. Ryabtsev, A.N.; Kononov, E.Y.; Kildiyarova, R.R.; Tchang-Brillet, W.-Ü.L.; Wyart, J.-F. $4 f^{13} 5 s^{2} 5 p^{6}-4 f^{13} 5 s^{2} 5 p^{5} 6 s$ transitions in the W VIII spectrum and spectra of isoelectronic hafnium, tantalum, and rhenium ions. Opt. Spectrosc. 2012, 113, 109-114.

16. Ryabtsev, A.N.; Kononov, E.Y.; Kildiyarova, R.R.; Tchang-Brillet, W.-Ü.L.; Wyart, J.-F. The spectrum of seven times ionized tungsten (W VII) relevant to tokamak divertor plasmas. Phys. Scr. 2013, 87, 045303.

17. Veres, G.; Bakos, J.S.; Kardon, B. Energy levels and the vacuum ultraviolet spectrum of W VIII. J. Quant. Spectrosc. Radiat. Transf. 1996, 56, 295-301.

18. Clementson, J.; Beiersdorfer, P.; Magee, E.W.; McLean, H.S.; Wood, R.D. Tungsten spectroscopy relevant to the diagnostic of ITER divertor plasmas. J. Phys. B At. Mol. Opt. Phys. 2010, 43, 144009.

19. Biedermann, C.; Radtke, R.; Schwob, J.-L.; Mandelbaum, P.; Doron, R.; Fuchs, T.; Fußmann, G. EUV spectroscopy of highly charged tungsten ions relevant to hot plasmas. Phys. Scr. 2001, 2001, 85-88.

20. Radtke, R.; Biedermann, C.; Schwob, J.L.; Mandelbaum, P.; Doron, R. Line and band emission from tungsten ions with charge $21+$ to $45+$ in the $45-70-\AA$ range. Phys. Rev. A 2001, 64, 012720.

21. Biedermann, C.; Radtke, R.; Seidel, R.; Pütterich, T. Spectroscopy of highly charged tungsten ions relevant to fusion plasmas. Phys. Scr. 2009, 2009, 014026.

22. Ralchenko, Y.; Reader, J.; Pomeroy, J.M.; Tan, J.N.; Gillaspy, J.D. Spectra of $\mathrm{W}^{39+}-\mathrm{W}^{4+}$ in the 12-20 nm region observed with an EBIT light source. J. Phys. B At. Mol. Opt. Phys. 2007, 40, 3861-3875.

23. Ralchenko, Y.; Draganić, I.N.; Tan, J.N.; Gillaspy, J.D.; Pomeroy, J.M.; Reader, J.; Feldman, U.; Holland, G.E. EUV spectra of highly-charged ions $\mathrm{W}^{54+}-\mathrm{W}^{63+}$ relevant to ITER diagnostics. J. Phys. B At. Mol. Opt. Phys. 2008, 41, 021003.

24. Ralchenko, Y.; Draganić, I.N.; Osin, D.; Gillaspy, J.D.; Reader, J. Spectroscopy of diagnostically important magnetic-dipole lines in highly charged $3 \mathrm{~d}^{n}$ ions of tungsten. Phys. Rev. A 2011, 83, 032517.

25. Fei, Z.; Zhao, R.; Shi, Z.; Xiao, J.; Qiu, M.; Grumer, J.; Andersson, M.; Brage, T.; Hutton, R.; Zou, Y. Experimental and theoretical study of the ground-state M1 transition in Ag-like tungsten. Phys. Rev. A 2012, 86, 062501.

26. Qiu, M.L.; Li, W.X.; Zhao, Z.Z.; Yang, Y.; Xiao, J.; Brage, T.; Hutton, R.; Zou, Y. Review of highly charged tungsten spectroscopy research using low energy EBIT at the Shanghai EBIT laboratory. J. Phys. B At. Mol. Opt. Phys. 2015, 48, 144029. 
27. Watanabe, H.; Nakamura, N.; Kato, D.; Nakano, T.; Ohtani, S. X-ray spectra from neon-like tungsten ions in the interaction with electrons. Plasma Fusion Res. 2007, 2, 027.

28. Sakaue, H.A.; Kato, D.; Ding, X.; Murakami, I.; Koike, F.; Nakano, T.; Yamamoto, N.; Ohashi, H.; Yatsurugi, J.; Nakamura, N. Spectroscopy of highly charged tungsten ions with electron beam ion traps. AIP Conf. Proc. 2012, 1438, 91-96.

29. Utter, S.B.; Beiersdorfer, P.; Brown, G.V. Measurement of an unusual M1 transition in the ground state of Ti-like $\mathrm{W}^{52+}$. Phys. Rev. A 2000, 61, 030503(R).

30. Clementson, J.; Beiersdorfer, P. Wavelength measurement of $n=3$ to $n=3$ transitions in highly charged tungsten ions. Phys. Rev. A 2010, 81, 052509.

31. Clementson, J.; Beiersdorfer, P.; Brown, G.V.; Gu, M.F.; Lundberg, H.; Podpaly, Y.; Träbert, E. Tungsten spectroscopy at the Livermore electron beam ion trap facility. Can. J. Phys. 2011, 89, 571-581.

32. Beiersdorfer, P.; Lepson, J.K.; Schneider, M.B.; Bode, M.P. L-shell X-ray emission from neonlike $\mathrm{W}^{64+}$. Phys. Rev. A 2012, 86, 012509.

33. Lennartsson, T.; Clementson, J.; Beiersdorfer, P. Experimental wavelengths for intrashell transitions in tungsten ions with partially filled 3p and 3d subshells. Phys. Rev. A 2013, 87, 062505.

34. Lepson, J.K.; Beiersdorfer, P. Low-energy operations of the Lawrence Livermore electron beam ion traps: Atomic spectroscopy of Si v, S VII and Ar IX. Phys. Scr. 2005, 2005, 62-65.

35. Levine, M.A.; Marrs, R.E.; Henderson, J.R.; Knapp, D.A.; Schneider, M.B. The electron beam ion trap: A new instrument for atomic physics measurements. Phys. Scr. 1988, 1988, 157-163.

36. Levine, M.A.; Marrs, R.E.; Bardsley, J.N.; Beiersdorfer, P.; Bennett, C.L.; Chen, M.H.; Cowan, T.; Dietrich, D.; Henderson, J.R.; Knapp, D.A.; et al. The use of an electron beam ion trap in the study of highly charged ions. Nucl. Instrum. Meth. B 1989, 43, 431-440.

37. Beiersdorfer, P.; Behar, E.; Boyce, K.R.; Brown, G.V.; Chen, H.; Gendreau, K.C.; Graf, A.; Gu, M.-F.; Harris, C.L.; Kahn, S.M.; et al. Overview of the Livermore electron beam ion trap project. Nucl. Instrum. Meth. B 2003, 205, 173-177.

38. Kelly, R.L. Atomic and ionic spectrum lines below 2000 Angstroms: Hydrogen through krypton. J. Phys. Chem. Ref. Data 1987, 16, 1-1678.

39. Bromander, J. The spectrum of triply-ionized oxygen, O IV. Ark. Fys. 1969, 40, 257-274.

40. Bockasten, K.; Johansson, K.B. Ark. Fys. 1968, 38, 563-584.

41. Edlén, B. The oxygen spectrum below $200 \AA$ and the high-limit terms of O IV. Phys. Scr. 1975, $11,366-370$.

42. Beiersdorfer, P.; Crespo López-Urrutia, J.R.; Springer, P.; Utter, S.B.; Wong, K.L. Spectroscopy in the extreme ultraviolet on an electron beam ion trap. Rev. Sci. Instrum. 1999, 70, 276-279.

43. Graf, A.T.; Brockington, S.; Horton, R.; Howard, S.; Hwang, D.; Beiersdorfer, P.; Clementson, J.; Hill, D.; May, M.; Mclean, H.; et al. Spectroscopy on magnetically confined plasmas using electron beam ion trap spectrometers. Can. J. Phys. 2008, 86, 307-313.

44. Clementson, J.; Beiersdorfer, P.; Magee, E.W. Grazing-incidence spectrometer on the SSPX spheromak. Rev. Sci. Instrum. 2008, 79, 10F538. 
45. Lepson, J.K.; Beiersdorfer, P.; Clementson, J.; Gu, M.F.; Bitter, M.; Roquemore, L.; Kaita, R.; Cox, P.G.; Safronova, A.S. EUV spectroscopy on NSTX. J. Phys. B At. Mol. Opt. Phys. 2010, 43, 144018.

46. Clementson, J.; Beiersdorfer, P.; Roquemore, A.L.; Skinner, C.H.; Mansfield, D.K.; Hartzfeld, K.; Lepson, J.K. Experimental setup for tungsten transport studies at the NSTX tokamak. Rev. Sci. Instrum. 2010, 81, 10E326.

47. Majeski, R.; Abrams, T.; Boyle, D.; Granstedt, E.; Hare, J.; Jacobson, C.M.; Kaita, R.; Kozub, T.; LeBlanc, B.; Lundberg, D.P.; et al. Particle control and plasma performance in the Lithium Tokamak eXperiment. Phys. Plasmas 2013, 20, 056103.

48. Beiersdorfer, P.; Magee, E.W.; Träbert, E.; Chen, H.; Lepson, J.K.; Gu, M.-F.; Schmidt, M. Flat-field grating spectrometer for high-resolution soft X-ray and extreme ultraviolet measurements on an electron beam ion trap. Rev. Sci. Instrum. 2004, 75, 3723-3726.

49. Beiersdorfer, P.; Magee, E.W.; Brown, G.V.; Hell, N.; Träbert, E.; Widmann, K. Extended-range grazing-incidence spectrometer for high-resolution extreme ultraviolet measurements on an electron beam ion trap. Rev. Sci. Instrum. 2014, 85, 11E422.

50. Safronova, U.I. (University of Nevada, USA). Personal Communications, 2010, 2011.

51. Safronova, U.I.; Safronova, A.S.; Beiersdorfer, P. Excitation energies, radiative and autoionization rates, dielectronic satellite lines, and dielectronic recombination rates for excited states of Yb-like W. J. Phys. B At. Mol. Opt. Phys. 2012, 45, 085001.

52. Cowan, R.D. The Theory of Atomic Structure and Spectra; University of California Press: Berkeley, CA, USA, 1981.

53. Skinner, C.H. Atomic physics in the quest for fusion energy and ITER. Phys. Scr. 2009, 2009, 014022.

54. Wenzel, U. (Max Planck Institute for Plasma Physics, Germany). Personal Communication, 2014.

55. Barnard, H.S.; Lipschultz, B.; Whyte, D.G. A study of tungsten migration in the Alcator C-Mod divertor. J. Nucl. Mater. 2011, 415, S301-S304.

(c) 2015 by the authors; licensee MDPI, Basel, Switzerland. This article is an open access article distributed under the terms and conditions of the Creative Commons Attribution license (http://creativecommons.org/licenses/by/4.0/). 